\title{
EGFR and HER2 signals play a salvage role in MEK1-mutated gastric cancer after MEK inhibition
}

\author{
TAKURO MIZUKAMI ${ }^{1,2}$, YOSUKE TOGASHI ${ }^{1}$, SHUNSUKE SOGABE $^{1}$, ERI BANNO ${ }^{1}$, MASATO TERASHIMA ${ }^{1}$, \\ MARCO A DE VELASCO ${ }^{1}$, KAZUKO SAKAI ${ }^{1}$, YOSHIHIKO FUJITA ${ }^{1}$, SHUTA TOMIDA ${ }^{1}$, \\ TAKAKO EGUCHI NAKAJIMA ${ }^{2}$, NARIKAZU BOKU ${ }^{2}$ and KAZUTO NISHIO ${ }^{1}$ \\ ${ }^{1}$ Department of Genome Biology, Kinki University School of Medicine, Osaka-Sayama, Osaka; \\ ${ }^{2}$ Department of Clinical Oncology, St. Marianna University School of Medicine, Kawasaki, Kanagawa, Japan
}

Received April 15, 2015; Accepted June 2, 2015

DOI: $10.3892 /$ ijo.2015.3050

\begin{abstract}
Since the prognosis of unresectable advanced gastric cancer remains poor, novel therapeutic strategies are needed. Somatic MEK1 gene mutations have been reported as oncogenic activating mutations in gastric cancer, and MEK inhibitors can be effective against such gastric cancers. In the present study, however, activated EGFR and HER2 signals after treatment with a MEK inhibitor (trametinib) were found in a MEK1-mutated gastric cancer cell line (OCUM-1 cell line) using a phospho-receptor tyrosine kinase array. The phosphorylation of EGFR and HER2 reactivated ERK1/2, which had been inhibited by trametinib, and EGF stimulation led to resistance to trametinib in this cell line. Lapatinib, an EGFR and an HER2 inhibitor, reversed the activation of ERK1/2 by inhibiting the phosphorylation of EGFR and HER2 and cancelled the resistance. The combination of trametinib and lapatinib synergistically inhibited the cell growth of the OCUM-1 cell line and strongly induced apoptosis by inhibiting the activated EGFR and HER2 signals. These results suggest that the EGFR and HER2 signals play a salvage role and are related to resistance to MEK inhibitors in MEK1-mutated gastric cancer. Moreover, combination therapy with trametinib and lapatinib can exhibit a synergistic effect and may contribute to overcoming the resistance to MEK inhibitors.
\end{abstract}

\section{Introduction}

Gastric cancer (GC) is one of the most common malignant tumors and the third most common cause of death from malignancies worldwide (1). Despite intensive investigations of anticancer treatments for GC, the prognosis of unresect-

Correspondence to: Professor Kazuto Nishio, Department of Genome Biology, Kinki University School of Medicine, 377-2 Ohnohigashi, Osaka-Sayama, Osaka 589-8511, Japan

E-mail:knishio@med.kindai.ac.jp

Key words: gastric cancer, MEK1 mutation, MEK inhibitor, EGFR, HER2 able advanced or recurrent $\mathrm{GC}$ remains poor. The median overall survival of GC patients has reached only $\sim 1$ year using conventional cytotoxic chemotherapy (2-4). Trastuzumab, a monoclonal antibody against human epidermal growth factor receptor (EGFR) 2 (HER2), used in combination with chemotherapy has shown a survival benefit when used as a first-line treatment in patients with HER2-positive advanced GC (5). However, HER2 overexpression has only been reported in 13-23\% of GC cases (6-8). Fibroblast growth factor receptor 2 amplification, MET amplification and RhoA mutations have been anticipated as new molecular targets in GC, but these aberrations are relatively infrequent (9-12). Therefore, new therapeutic modalities are still needed.

The mitogen-activated protein kinase (MAPK) signal pathway cascade and its downstream factors promote cancer cell proliferation, differentiation and survival. The three-tiered kinase cascade consisting of RAF, mitogen-activated protein kinase kinase (MEK), and extracellular signal-regulated kinase (ERK) is frequently dysregulated in malignancies as a result of activating mutations in the upstream $R A S$ (KRAS, $N R A S$ and $H R A S)(13,14)$. The activating $B R A F$ mutation has been detected in a variety of human cancers (15), and the success of RAF inhibitors in $B R A F$-mutated melanoma has revealed a new modality and has substantiated the contribution of the MAPK signal to carcinogenesis $(16,17)$. Somatic oncogenic MEK1 mutations have also been identified in several human malignancies (18-21). In addition, our previous study demonstrated that $M E K 1$ mutations in poorly differentiated GC cell lines that are hypersensitive to MEK inhibitors have transformational abilities and that the growth of these cancer cells is dependent on these mutations (22). Considering the addiction of cancer cells to active $M E K 1$ mutations for proliferation, GC with such oncogenic $M E K 1$ mutations might be suitable for targeted therapy with MEK inhibitors.

A persistent problem is the development of resistance to molecular-targeted therapies. A series of resistance mechanisms for MEK inhibition that operate ERK-dependently or ERK-independently have been reported, including $M E K$ mutations, elevated RAS or RAF protein levels, and the activation of an alternative PI3K/AKT or STAT3 pathway (23-25). Currently, the phosphorylation of EGFR after the inhibition of the MAPK signal has been suggested as another potential 
mechanism responsible for the resistance to MEK inhibition in various cancers, and combination therapy with EGFR inhibitors yielded synergistic effects $(26,27)$. In this study, we observed the phosphorylation of EGFR and HER2 after MEK inhibition in a MEK1-mutated GC cell line. Then, the relevancy of this mechanism to MEK inhibitor resistance was validated, and combinations with other tyrosine kinase inhibitors were tested to develop new therapeutic possibilities.

\section{Materials and methods}

Reagents and ligand. Trametinib (GSK1120212) and lapatinib (GW572016) were purchased from Selleck Chemicals (Houston, TX, USA) and were dissolved in dimethyl sulfoxide (DMSO) for the in vitro experiments. Recombinant human epidermal growth factor (EGF) was obtained from R\&D Systems (Minneapolis, MN, USA) and was constituted in sterile phosphate-buffered saline (PBS).

Antibodies. Rabbit-antibodies specific for ERK1/2, EGFR, HER2, phospho-ERK1/2 (pERK1/2), phospho-EGFR (pEGFR), phospho-HER2 (pHER2), poly (ADP-ribose) polymerase (PARP), caspase-3, cleaved PARP (cPARP), cleaved caspase-3 (cCaspase-3), and $\beta$-actin were obtained from Cell Signaling Technology (Beverly, MA, USA).

Cell cultures. A poorly differentiated GC cell line harboring the MEK1 Q56P mutation, OCUM-1, was propagated in RPMI1640 (Sigma-Aldrich, St. Louis, MO, USA) supplemented with $10 \%$ heat-inactivated fetal bovine serum (FBS; Gibco BRL, Grand Island, NY, USA) and 1\% Penicillin-Streptomycin Mixed Solution (Nacalai Tesque, Kyoto, Japan) in a humidified atmosphere of $5 \% \mathrm{CO}_{2}$ at $37^{\circ} \mathrm{C}$.

Human phospho-receptor tyrosine kinase (RTK) array. The screening of 49 phosphorylated tyrosine kinases in the OCUM-1 cell line was performed using the Human Phospho-RTK Array kit (R\&D Systems). Whole lyses of OCUM-1 cells incubated in the absence (DMSO) or presence of $1 \mathrm{nM}$ trametinib for $72 \mathrm{~h}$ were compared in accordance with the manufacturer's recommendation. Protein detection was accomplished using an anti-phospho-tyrosine-HRP detection antibody and an enhanced chemiluminescence system (Image Quant LAS4000; GE Healthcare Life Science, Buckinghamshire, UK).

Growth inhibition assay. The 3-(4,5-dimethylthiazol-2-yl)2,5-diphenyltetrazolium bromide (MTT) assay was used to evaluate the growth inhibitory effect of the drugs, as previously described (28). When the effect of EGF was evaluated, $1 \%$ FBS was used.

Real-time reverse transcription polymerase chain reaction $(R T-P C R)$. A total of $1 \mu \mathrm{g}$ of RNA was isolated from the cells using ISOGEN reagent (Nippon Gene, Tokyo, Japan) and then converted to cDNA using GeneAmp RNA-PCR kit (Applied Biosystems, Foster City, CA USA). Real-time PCR was performed using SYBR Premix Ex Taq and Thermal Cycler Dice (Takara, Shiga, Japan), as described previously (29). The glyceraldehyde 3-phosphate dehydrogenase (GAPD,
NM_002046) gene was used to normalize the expression levels in subsequent quantitative analyses. To amplify the target genes encoding amphiregulin (AREG), EGF, heparinbinding EGF-like growth factor (HB-EGF), neuregulin 1 (NRG1), transforming growth factor $\alpha$ (TGFA), EGFR, HER2, HER3, and HER4 (AREG, EGF, HB-EGF, NRG1, TGFA, $E G F R, E R B B 2, E R B B 3$ and $E R B B 4$ genes, respectively), the following primers were used: AREG-F, GTCGCTCTTGATAC TCGGCTCAG; AREG-R, TCCCAGAGTAGGTGTCATTG AGGTC; EGF-F, CAACCAGTGGCTGGTGAGGA; EGF-R, GAGCCCTTATCACTGGATACTGGAA; HB-EGF-F, CAA GGTGATTTCAGACTGCAGAGG; HB-EGF-R, TTTGGCA CTTGAAGGCTCTGG; NRG1-F, GCCAGGAATCGGCTG CAGGT; NRG1-R, AGCCAGTGATGCT TTGTTAATGCGA; TGFA-F, CTTTGGAAACCAGCAGGTCTGA; TGFA-R, CCCAAATAAGCCAGGCTGTTCTA; EGFR-F, CATCCA GGCCCAACTGTGAG; EGFR-R, CAGTGGAAGCCTT GAAGCAGAA; ERBB2-F, TGGGAGCCTGGCATTTCTG; ERBB2-R, CGGCCATGCTGAGATGTATAGGTA; ERBB3-F, GGGAGCATTTAATGGCAGCTA; ERBB3-R, GAATGGAATTGTCTGGGACTGG; ERBB4-F, GCAGCT AACTTTGAATGCCTGTCTC; ERBB4-R, GCAGCTA ACTTTGAATGCCTGTCTC; GAPD-F, GCACCGTCAAG GCTGAGAAC; and GAPD-R, ATGGTGGTGAAGACG CCAGT.

Western blot analysis. The western blot analysis was performed as described previously (28). Briefly, subconfluent cells were washed with cold PBS and harvested with Lysis A buffer containing 1\% Triton X-100, $20 \mathrm{mM}$ Tris- $\mathrm{HCl}(\mathrm{pH} 7.0), 5 \mathrm{mM}$ EDTA, $50 \mathrm{mM}$ sodium chloride, $10 \mathrm{mM}$ sodium pyrophosphate, $50 \mathrm{mM}$ sodium fluoride, $1 \mathrm{mM}$ sodium orthovanadate, and a protease inhibitor mix, Complete ${ }^{\mathrm{TM}}$ (Roche Diagnostics). Whole-cell lyses were separated using SDS-PAGE and were blotted onto a polyvinylidene fluoride membrane. After blocking with $3 \%$ bovine serum albumin in a TBS buffer (pH 8.0) with $0.1 \%$ Tween-20, the membrane was probed with the primary antibody. After rinsing twice with TBS buffer, the membrane was incubated with a horseradish peroxidaseconjugated secondary antibody and washed, followed by visualization using an ECL detection system and LAS-4000. When the cells were stimulated using EGF, the cells were incubated with $1 \%$ FBS medium.

Statistical analysis. The results are shown as the mean value \pm standard deviation $(\mathrm{SD})$ for three replicate independent experiments. The statistical analyses were performed using the Student's t-test (two-tailed). A P-value $<0.05$ was considered statistically significant. The statistical analyses were two-tailed and were performed using Microsoft Excel (Microsoft, Redmond, WA, USA).

\section{Results}

$M E K$ inhibition led to the phosphorylation of EGFR and HER2 in the MEK1-mutated OCUM-1 cell line. To address the phosphorylation of RTK after MEK inhibition, we used a phospho-RTK array. The arrangement of the RTK array is summarized in Fig. 1B. The phosphorylation levels of EGFR and HER2 were elevated at $72 \mathrm{~h}$ after treatment with a MEK 
$\mathbf{A}$

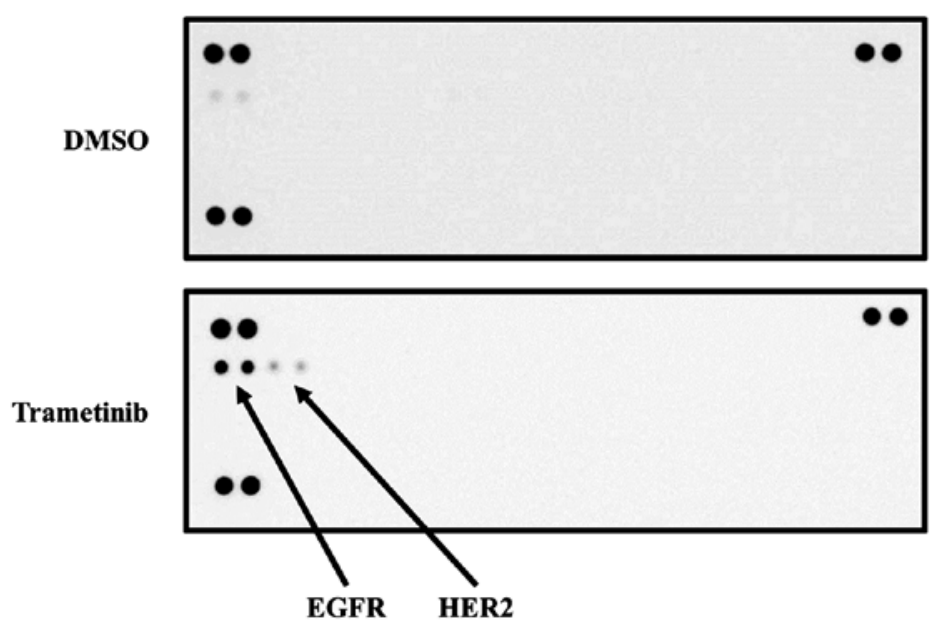

B

\begin{tabular}{|c|c|c|c|c|c|c|c|c|c|c|c|}
\hline Ref. & & & & & & & & & & & Ref. \\
\hline EGFR & HER2 & HER3 & HER4 & FGFR1 & FGFR2 $\alpha$ & FGFR3 & FGFR4 & Insulin R & IGF-I R & Axl & Dtk \\
\hline Mer & HGFR & MSPR & PDGFR $\alpha$ & PDGFR $\beta$ & SCFR & Flt-3 & M-CSFR & C-Ret & ROR1 & ROR2 & Tie-1 \\
\hline Tie-2 & TrkA & TrkB & TrkC & VEGFR1 & VEGFR2 & VEGFR3 & MuSK & EphA1 & EphA2 & EphA3 & EphA4 \\
\hline EphA6 & EphA7 & EphB1 & EphB2 & EphB4 & EphB6 & ALK & DDR1 & DDR2 & EphA5 & EphA10 & \\
\hline Ref. & & EphB3 & RYK & & & & & & & & PBS \\
\hline
\end{tabular}

Figure 1. Phosphorylation of RTKs after MEK inhibition in the OCUM-1 cell line. (A) Phospho-RTKs in MEK1-mutated OCUM-1 cells incubated in the absence (DMSO) or presence of $1 \mathrm{nM}$ of trametinib for $72 \mathrm{~h}$ were compared using a human phospho-RTK array. Each pair of horizontal spots represents one RTK. The densities of two pairs of spots, representing the phosphorylation levels of EGFR and HER2, were greatly increased after MEK inhibition with trametinib. (B) Arrangement of phospho-RTK array. The paired spots in 3 of the 4 corners are phosphorylated tyrosine controls used as references, and the spots in the lower right corner represent PBS, which was used as a negative control.

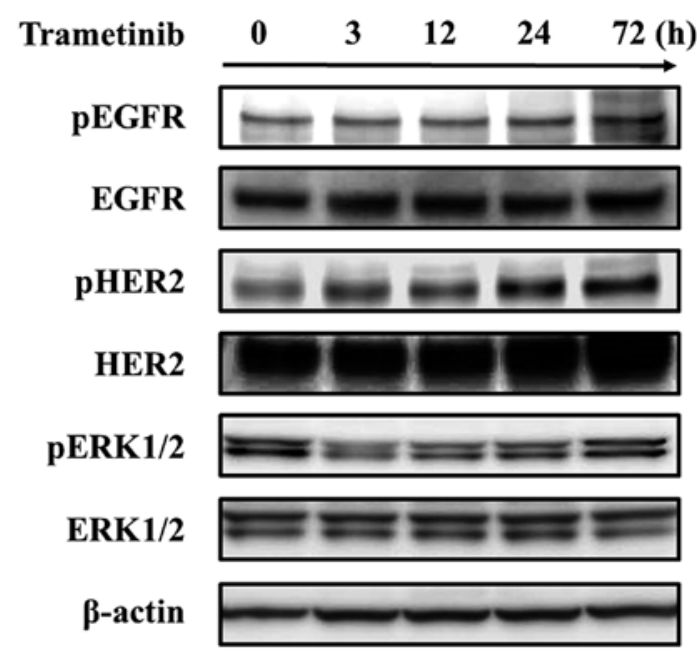

Figure 2. Time course for the phosphorylation levels of EGFR, HER2 and ERK1/2 after MEK inhibition. The OCUM-1 cells were incubated with $1 \mathrm{nM}$ of trametinib for each of the indicated time periods and were then evaluated using a western blot analysis. The phosphorylation levels of EGFR and HER 2 were increased at 24 and $72 \mathrm{~h}$ after trametinib exposure. Trametinib antecedently inhibited the phosphorylation levels of ERK1/2 from $3 \mathrm{~h}$, and inhibited ERK1/2 was re-activated in accordance with the elevation of the phosphorylation levels of EGFR and HER2. $\beta$-actin was used as an internal control. pEGFR, phospho-EGFR; pHER2, phosphoHER2; pERK1/2, phospho-ERK1/2. inhibitor (trametinib) (Fig. 1A). Next, to validate the results of the RTK array, we evaluated the phosphorylation level of EGFR and HER2 using a western blot analysis. In addition to the inhibitory effect on the ERK signal, trametinib led to the activation of EGFR and HER2 signals similar to the results of the RTK array. In addition, the phosphorylation of ERK1/2, which had been inhibited by trametinib, was reactivated following the activation of the EGFR and HER2 signals (Fig. 2). These results suggest that the EGFR and HER2 signals are activated under MEK inhibition in MEK1-mutated GC. To investigate the mechanism responsible for the activation of the EGFR and HER2 signals, real-time RT-PCR was performed. However, no significant changes in the mRNA expression levels of the molecules relative to the EGFR and HER2 signals were observed (AREG, EGF, HB-EGF, NRGI, TGFA, EGFR, HER2, HER3 and HER4; data not shown).

Ligand-induced EGFR and HER2 activation led to the acquisition of resistance to a MEK inhibitor. To verify the contribution of EGFR and HER2 signals to MEK inhibitor resistance, the experiments were performed using recombinant human EGF (10 ng/ml) as the ligand. After the EGF-induced activation of the EGFR and HER2 signals, the OCUM-1 cell line became resistant to trametinib (Fig. 3A). Using a western 
A

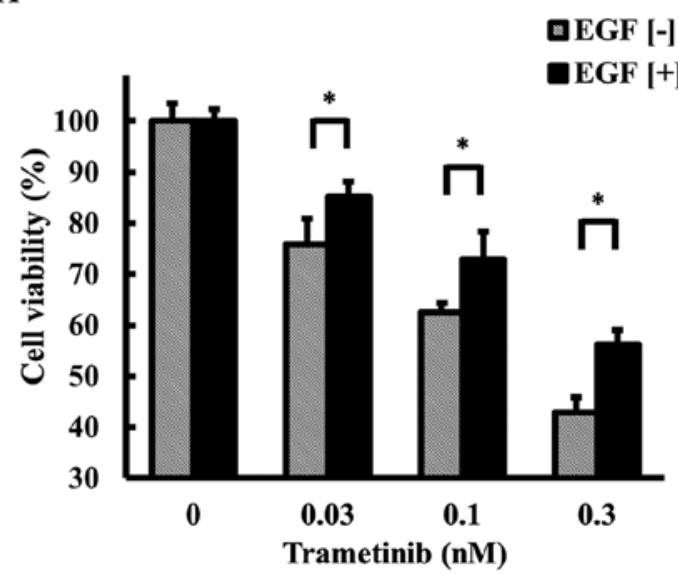

B

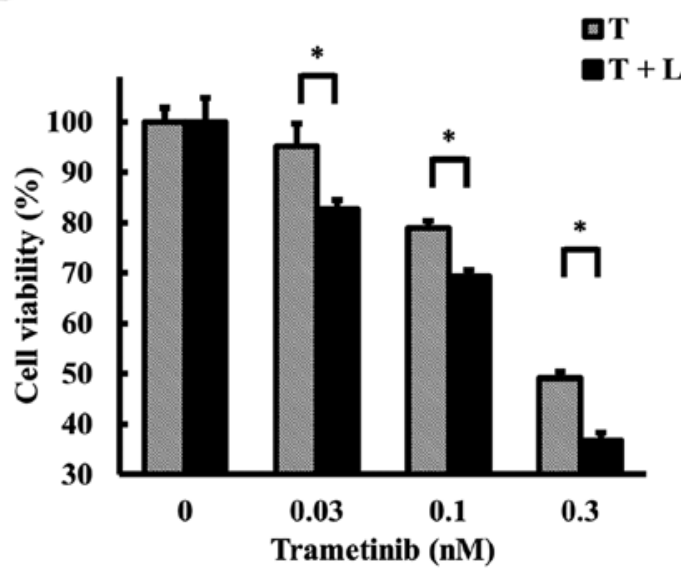

Figure 3. Growth inhibitory effect of trametinib in the OCUM-1 cell line. (A) Influence of EGF on the sensitivity to trametinib. Cells were exposed to each concentration of trametinib for $72 \mathrm{~h}$ with or without $10 \mathrm{ng} / \mathrm{ml}$ of EGF [EGF (+), solid bars; EGF (-), bars with hatch lines] and were evaluated using an MTT assay using $1 \%$ FBS. The growth inhibitory effect at every concentration of trametinib was strongly weakened by EGF stimulation. Columns, mean of independent triplicate experiments; error bars, SD; ${ }^{*} \mathrm{P}<0.05$. (B) Growth inhibitory effect of trametinib monotherapy and combination with lapatinib. The cells were exposed to each concentration of trametinib for $72 \mathrm{~h}$ with or without $10 \mu \mathrm{M}$ of lapatinib $(\mathrm{T}+\mathrm{L}$, solid bars; $\mathrm{T}$, bars with hatch lines) and were evaluated using an MTT assay. The combination of trametinib and lapatinib synergistically inhibited the cell growth in a significant manner. Columns, mean of independent triplicate experiments; error bars, SD; $\mathrm{P}<0.05$.

A

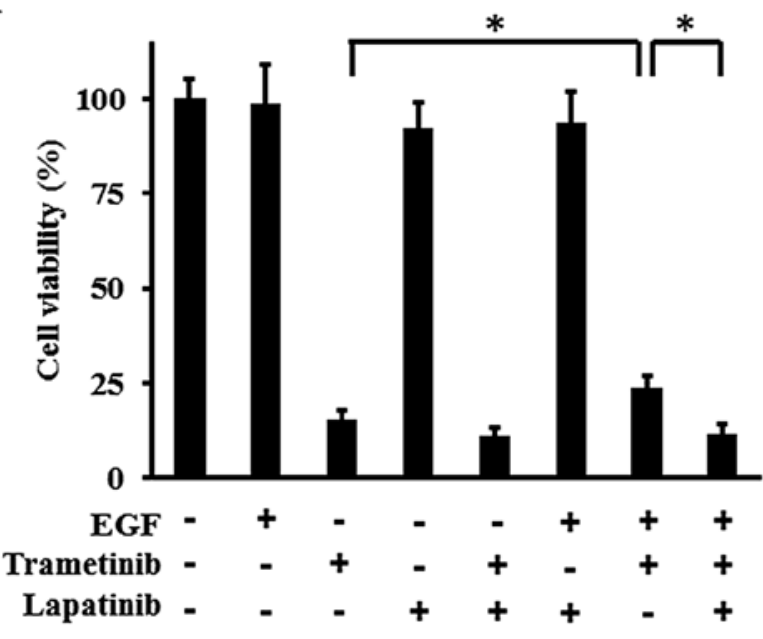

B

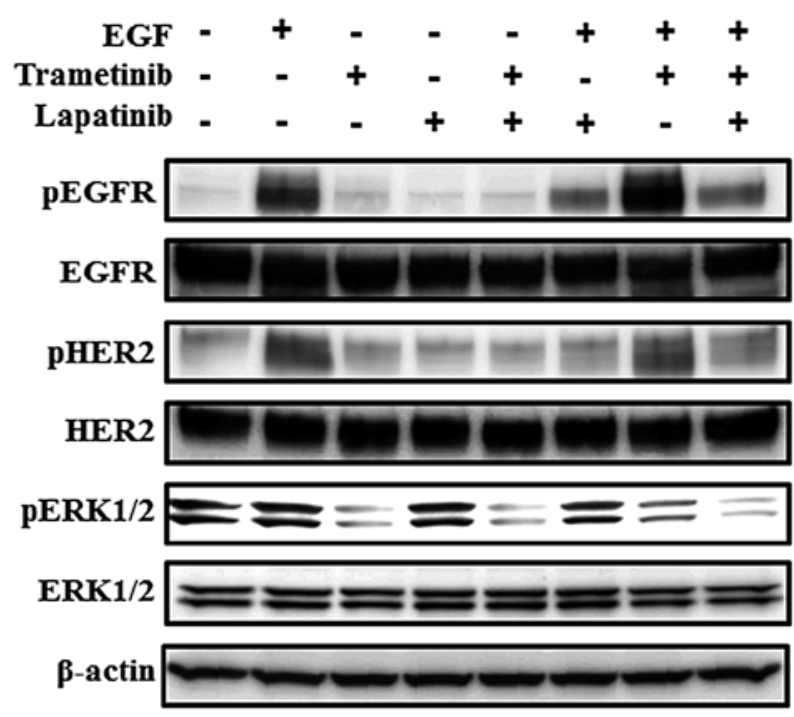

Figure 4. Effect of lapatinib on the resistance to trametinib induced by EGF. (A) Growth inhibitory effect of combination with trametinib and lapatinib in the presence of EGF. The cells were exposed to $1 \mathrm{nM}$ of trametinib, $10 \mu \mathrm{M}$ of lapatinib, and/or $10 \mathrm{ng} / \mathrm{ml}$ of EGF for $72 \mathrm{~h}$ and the cell growth was evaluated using an MTT assay with 1\% FBS. In the presence of EGF, the inhibitory effect of trametinib was weakened significantly, while the combination of trametinib and lapatinib abolished the resistance induced by EGF. EGF or lapatinib monotherapy did not influence the cell growth. Columns, mean of independent triplicate experiments; error bars, SD; "P<0.05. (B) Phosphorylation of EGFR, HER2, and ERK1/2. When the phosphorylation levels were examined using a western blot analysis, the samples were collected $3 \mathrm{~h}$ after stimulation. The following concentrations were used: $1 \mathrm{nM}$ of trametinib, $10 \mu \mathrm{M}$ of lapatinib, and $10 \mathrm{ng} / \mathrm{ml}$ of EGF. EGF stimulation increased the phosphorylation levels of EGFR and HER2, resulting in the activation of ERK1/2. The decreased phosphorylation levels of ERK1/2 induced by trametinib were re-activated by EGF stimulation, and lapatinib abolished the re-activation of ERK1/2 by inhibiting the phosphorylation of EGFR and HER2. $\beta$-actin was used as an internal control. pEGFR, phospho-EGFR; pHER2, phospho-HER2; pERK1/2, phospho-ERK1/2.

blot analysis, EGF stimulation was shown to increase the phosphorylation levels of EGFR and HER2, and ERK1/2, which had been suppressed by trametinib, was re-phosphorylated (Fig. 4B).

Lapatinib abolished the resistance to trametinib induced by $E G F$. Next, to investigate the effect of lapatinib (an EGFR and
HER2 dual tyrosine kinase inhibitor) on the EGF-induced resistance, a combination therapy was tested. The EGF-induced resistance to trametinib was abolished by lapatinib (Fig. 4A). The suppression of the phosphorylation levels of ERK $1 / 2$ through the inhibition of EGFR and HER2 by lapatinib can explain the restored response to trametinib (Fig. 4B). These results suggest that the activation of EGFR and HER2 signals 
A

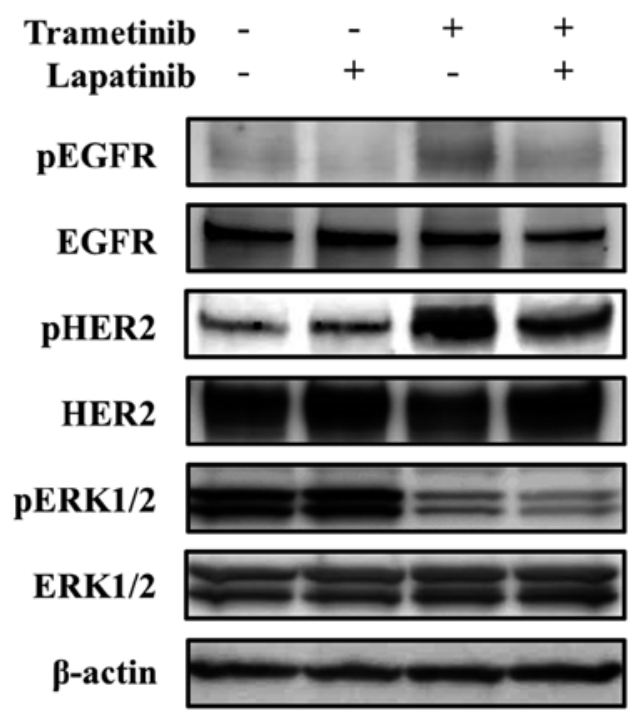

B

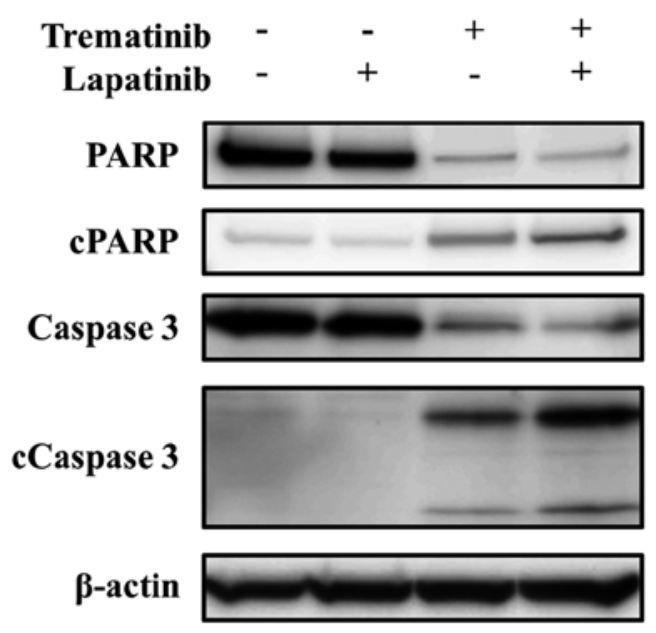

Figure 5. Synergistic effect of trametinib and lapatinib on EGFR, HER2, and ERK signals and apoptosis-related molecules. (A) Phosphorylation of EGFR, HER2, and ERK1/2. For the analysis of the phosphorylation levels using a western blot analysis, the samples were collected at $72 \mathrm{~h}$ after exposure. The following concentrations were used: $1 \mathrm{nM}$ of trametinib and $10 \mu \mathrm{M}$ of lapatinib. The phosphorylation levels of EGFR and HER2 were elevated at $72 \mathrm{~h}$ after treatment with trametinib, similar to the results obtained using the phospho-RTK array. Lapatinib reduced the phosphorylation level of ERK1/2 by inhibiting the EGFR and HER2 signals, which were activated after trametinib treatment. $\beta$-actin was used as an internal control. pEGFR, phospho-EGFR; pHER2, phospho-HER2; pERK1/2, phospho-ERK1/2. (B) Expressions of apoptosis-related molecules. The cells were exposed to the inhibitors for 72 h, and apoptosisrelated molecules were then examined using a western blot analysis. The following concentrations were used: $1 \mathrm{nM}$ of trametinib and $10 \mu \mathrm{M}$ of lapatinib. Trametinib increased the expressions of cleaved PARP and cleaved caspase- 3 to a greater extent when used in combination with lapatinib, compared with the results for trametinib monotherapy. $\beta$-actin was used as an internal control. cPARP, cleaved PARP; cCaspase-3, cleaved caspase-3.

potentially plays an important role in MEK inhibitor resistance and that lapatinib may abolish such resistance.

Combination of trametinib and lapatinib synergistically inhibits cell growth. To investigate the role of the activated EGFR and HER2 signals during treatment with trametinib, we examined the synergistic anticancer effect of trametinib and lapatinib in the OCUM-1 cell line using an MTT assay. The combination of trametinib and lapatinib showed a synergistic effect on the inhibition of cell proliferation (Fig. 3B). Furthermore, lapatinib decreased the phosphorylation levels of EGFR and HER2, which had been activated after the treatment with trametinib, and the phosphorylation level of ERK1/2 was inhibited to a greater degree by the combination treatment than by trametinib monotherapy (Fig. 5A). Cleaved PARP and cleaved caspase-3, which are hallmarks of apoptosis, had also increased at $72 \mathrm{~h}$ after the trametinib and lapatinib combination therapy, compared with after trametinib monotherapy (Fig. 5B). These results suggest that activated EGFR and HER2 signals are involved in salvage pathways under MEK inhibition in MEK1-mutated GC and that trametinib and lapatinib exert a synergistic effect by inhibiting the salvage signals.

\section{Discussion}

In this study, we demonstrated the reactivation of ERK1/2 via the activation of EGFR and HER2 signals after MEK inhibition with trametinib, and EGF-induced resistance in the MEK1-mutated GC cell line. Our data also identified the receptor tyrosine kinase family members EGFR and HER2 as potent targets for overcoming resistance to MEK inhibi- tors. Moreover, the combination of trametinib and lapatinib was more effective against a $M E K 1$-mutated GC cell line than trametinib monotherapy. To the best of our knowledge, this is the first report to show the activation of EGFR and HER2 after MEK inhibition, the potential of combination therapy as a therapeutic strategy, and the possibility of overcoming resistance in MEK1-mutated GC.

The serine threonine kinases BRAF, MEK, and ERK are major regulators of the MAPK signal and are frequently dysregulated in malignancies. Given the role of these kinases in cancer, the MAPK signal has become an attractive target for molecular targeted drugs (30). While a number of MAPK signal inhibitors, as well as RAF inhibitors, have been developed and are in clinical use, the acquisition of resistance has hindered the continuation of treatment, resulting in a limited survival benefit. Resistance to MAPK inhibitors is mediated by many mechanisms, including the reactivation of RAS/RAF/MEK/ERK signals, $M E K$ mutations, and increases in the RAS or RAF protein levels, as well as increased signals through alternative pathways, including the PI3K/AKT and STAT3 pathways (31). Recently, BRAF inhibition leading to a feedback activation of EGFR and a strong synergistic effect of BRAF and EGFR inhibition were described in $B R A F$-mutated colorectal cancer cell lines (32). Furthermore, the combination of a MEK inhibitor and a dual EGFR and HER2 inhibitor produced a synergistic effect by inhibiting the activation of ERK signals both in vivo and in vitro in $K R A S$-mutated colon and lung cancer cell lines (27). These results suggest that the activation of HER family members is an important salvage signal during MAPK inhibition. Similarly, in our data, the EGFR and HER2 signals were activated after treatment with 
a MEK inhibitor, resulting in the re-activation of the ERK signal. In addition, EGF stimulation also activated the ERK signal via the activation of EGFR and HER2 in the MEK1mutated GC cell line, which led to MEK inhibitor resistance. The effects of the EGFR and HER2 signals were abolished by the inhibitor. These findings indicate the possibility of a novel combination therapy comprised of a MEK inhibitor and a HER inhibitor for GC patients, similar to results observed for other malignancies.

To address how MEK inhibition by trametinib activates EGFR and HER2, we investigated the mRNA expression of the corresponding genes using real-time RT-PCR. However, no significant change in mRNA expression was observed. MEK inhibition reportedly leads to MYC degradation and HER2 and HER3 upregulation in KRAS-mutated colon and lung cancer (27). Another study has shown that CDC25c, which can bind to EGFR, may be involved in the activation of EGFR in a $B R A F$-mutated colorectal cancer cell line (32). However, the detailed mechanism responsible for the activation of HER signals after MEK inhibition remains unclear. Furthermore, the activation of HER signals has been reported to result not only from MAPK inhibition, but also from exposure to cytotoxic agents, including 5-FU, oxaliplatin in colorectal cancer cells, and pemetrexed in non-small cell lung cancer $(33,34)$. These findings suggest that the activation of HER signals plays a primary salvage role in many cancers, promising that resistance to many anticancer therapies can be overcome. Therefore, further research on approaches to overcoming such resistance is needed.

In conclusion, the present study revealed that the activation of EGFR and HER2 signals after MEK inhibition may serve as a potential mechanism responsible for the resistance to a MEK inhibitor in MEK1-mutated GC. Additionally, combined treatment with trametinib and lapatinib exhibited a synergistic effect by inhibiting the activation of the EGFR and HER2 signals. These data may contribute to the development of novel therapeutic strategies for MEK1-mutated GC.

\section{Acknowledgements}

We thank Mr. Shinji Kurashimo, Mr. Yoshihiro Mine, Ms. Eiko Honda, Ms. Tomoko Kitayama and Ms. Ayaka Kurumatani for their technical assistance. This study was supported in part by the Grant-in Aid for Japan Society for Promotion of Science Fellows.

\section{References}

1. Ferlay J, Soerjomataram I, Dikshit R, Eser S, Mathers C, Rebelo M, Parkin DM, Forman D and Bray F: Cancer incidence and mortality worldwide: Sources, methods and major patterns in GLOBOCAN 2012. Int J Cancer 136: E359-E386, 2015.

2. Van Cutsem E, Moiseyenko VM, Tjulandin S, Majlis A, Constenla M, Boni C, Rodrigues A, Fodor M, Chao Y, Voznyi E, et al; V325 Study Group: Phase III study of docetaxel and cisplatin plus fluorouracil compared with cisplatin and fluorouracil as first-line therapy for advanced gastric cancer: A report of the V325 Study Group. J Clin Oncol 24: 4991-4997, 2006.

3. Cunningham D, Starling N, Rao S, Iveson T, Nicolson M, Coxon F, Middleton G, Daniel F, Oates J and Norman AR; Upper Gastrointestinal Clinical Studies Group of the National Cancer Research Institute of the United Kingdom: Capecitabine and oxaliplatin for advanced esophagogastric cancer. N Engl J Med 358: 36-46, 2008.
4. Koizumi W, Narahara H, Hara T, Takagane A, Akiya T, Takagi M, Miyashita K, Nishizaki T, Kobayashi O, Takiyama W, et al: S-1 plus cisplatin versus $\mathrm{S}-1$ alone for first-line treatment of advanced gastric cancer (SPIRITS trial): A phase III trial. Lancet Oncol 9: 215-221, 2008.

5. Bang YJ, Van Cutsem E, Feyereislova A, Chung HC, Shen L, Sawaki A, Lordick F, Ohtsu A, Omuro Y, Satoh T, et al; ToGA Trial Investigators: Trastuzumab in combination with chemotherapy versus chemotherapy alone for treatment of HER2-positive advanced gastric or gastro-oesophageal junction cancer (ToGA): A phase 3, open-label, randomised controlled trial. Lancet 376: 687-697, 2010.

6. Sakai K, Mori S, Kawamoto T, Taniguchi S, Kobori O, Morioka Y, Kuroki T and Kano K: Expression of epidermal growth factor receptors on normal human gastric epithelia and gastric carcinomas. J Natl Cancer Inst 77: 1047-1052, 1986.

7. Yano T, Doi T, Ohtsu A, Boku N, Hashizume K, Nakanishi M and Ochiai A: Comparison of HER2 gene amplification assessed by fluorescence in situ hybridization and HER2 protein expression assessed by immunohistochemistry in gastric cancer. Oncol Rep 15: 65-71, 2006.

8. Gravalos C and Jimeno A: HER2 in gastric cancer: A new prognostic factor and a novel therapeutic target. Ann Oncol 19: 1523-1529, 2008

9. Matsumoto K, Arao T, Hamaguchi T, Shimada Y, Kato K, Oda I, Taniguchi H, Koizumi F, Yanagihara K, Sasaki H, et al: FGFR2 gene amplification and clinicopathological features in gastric cancer. Br J Cancer 106: 727-732, 2012.

10. Kawakami H, Okamoto I, Arao T, Okamoto W, Matsumoto K, Taniguchi H, Kuwata K, Yamaguchi H, Nishio K, Nakagawa K, et al: MET amplification as a potential therapeutic target in gastric cancer. Oncotarget 4: 9-17, 2013.

11. Wang K, Yuen ST, Xu J, Lee SP, Yan HH, Shi ST, Siu HC, Deng S, Chu KM, Law S, et al: Whole-genome sequencing and comprehensive molecular profiling identify new driver mutations in gastric cancer. Nat Genet 46: 573-582, 2014.

12. Kakiuchi M, Nishizawa T, Ueda H, Gotoh K, Tanaka A, Hayashi A, Yamamoto S, Tatsuno K, Katoh H, Watanabe Y, et al: Recurrent gain-of-function mutations of RHOA in diffuse-type gastric carcinoma. Nat Genet 46: 583-587, 2014.

13. Johnson GL and Lapadat R: Mitogen-activated protein kinase pathways mediated by ERK, JNK, and p38 protein kinases. Science 298: 1911-1912, 2002.

14. Roberts PJ and Der CJ: Targeting the Raf-MEK-ERK mitogenactivated protein kinase cascade for the treatment of cancer. Oncogene 26: 3291-3310, 2007.

15. Davies H, Bignell GR, Cox C, Stephens P, Edkins S, Clegg S, Teague J, Woffendin H, Garnett MJ, Bottomley W, et al: Mutations of the BRAF gene in human cancer. Nature 417: 949-954, 2002.

16. Bollag G, Hirth P, Tsai J, Zhang J, Ibrahim PN, Cho H, Spevak W, Zhang C, Zhang Y, Habets G, et al: Clinical efficacy of a RAF inhibitor needs broad target blockade in BRAF-mutant melanoma. Nature 467: 596-599, 2010.

17. Flaherty KT, Puzanov I, Kim KB, Ribas A, McArthur GA, Sosman JA, O'Dwyer PJ, Lee RJ, Grippo JF, Nolop K, et al: Inhibition of mutated, activated BRAF in metastatic melanoma. N Engl J Med 363: 809-819, 2010.

18. Pao W and Girard N: New driver mutations in non-small-cell lung cancer. Lancet Oncol 12: 175-180, 2011.

19. Estep AL, Palmer C, McCormick F and Rauen KA: Mutation analysis of BRAF, MEK1 and MEK2 in 15 ovarian cancer cell lines: Implications for therapy. PLoS One 2: e1279, 2007.

20. Marks JL, Gong Y, Chitale D, Golas B, McLellan MD, Kasai Y, Ding L, Mardis ER, Wilson RK, Solit D, et al: Novel MEK1 mutation identified by mutational analysis of epidermal growth factor receptor signaling pathway genes in lung adenocarcinoma. Cancer Res 68: 5524-5528, 2008.

21. Bentivegna S, Zheng J, Namsaraev E, Carlton VE, Pavlicek A, Moorhead M, Siddiqui F, Wang Z, Lee L, Ireland JS, et al: Rapid identification of somatic mutations in colorectal and breast cancer tissues using mismatch repair detection (MRD). Hum Mutat 29: 441-450, 2008.

22. Sogabe S, Togashi Y, Kato H, Kogita A, Mizukami T, Sakamoto Y, Banno E, Terashima M, Hayashi $\mathrm{H}$, de Velasco MA, et al: MEK inhibitor for gastric cancer with MEK1 gene mutations. Mol Cancer Ther 13: 3098-3106, 2014.

23. Emery CM, Vijayendran KG, Zipser MC, Sawyer AM, Niu L, Kim JJ, Hatton C, Chopra R, Oberholzer PA, Karpova MB, et al: MEK1 mutations confer resistance to MEK and B-RAF inhibition. Proc Natl Acad Sci USA 106: 20411-20416, 2009. 
24. Little AS, Balmanno K, Sale MJ, Newman S, Dry JR, Hampson M, Edwards PA, Smith PD and Cook SJ: Amplification of the driving oncogene, KRAS or BRAF, underpins acquired resistance to MEK1/2 inhibitors in colorectal cancer cells. Sci Signal 4: ra17, 2011.

25. Corcoran RB, Dias-Santagata D, Bergethon K, Iafrate AJ, Settleman J and Engelman JA: BRAF gene amplification can promote acquired resistance to MEK inhibitors in cancer cells harboring the BRAF V600E mutation. Sci Signal 3: ra84, 2010.

26. Walters DM, Lindberg JM, Adair SJ, Newhook TE, Cowan CR, Stokes JB, Borgman CA, Stelow EB, Lowrey BT, Chopivsky ME, et al: Inhibition of the growth of patient-derived pancreatic cancer xenografts with the MEK inhibitor trametinib is augmented by combined treatment with the epidermal growth factor receptor/ HER2 inhibitor lapatinib. Neoplasia 15: 143-155, 2013.

27. Sun C, Hobor S, Bertotti A, Zecchin D, Huang S, Galimi F, Cottino F, Prahallad A, Grernrum W, Tzani A, et al: Intrinsic resistance to MEK inhibition in KRAS mutant lung and colon cancer through transcriptional induction of ERBB3. Cell Rep 7: 86-93, 2014.

28. Togashi $Y$, Sakamoto $H$, Hayashi $H$, Terashima $M$, De Velasco MA, Fujita Y, Kodera Y, Sakai K, Tomida S, Kitano M, et al: Homozygous deletion of the activin A receptor, type IB gene is associated with an aggressive cancer phenotype in pancreatic cancer. Mol Cancer 13: 126, 2014.
29. Kaneda H, Arao T, Tanaka K, Tamura D, Aomatsu K, Kudo K, Sakai K, De Velasco MA, Matsumoto K, Fujita Y, et al: FOXQ1 is overexpressed in colorectal cancer and enhances tumorigenicity and tumor growth. Cancer Res 70: 2053-2063, 2010.

30. Hingorani SR, Jacobetz MA, Robertson GP, Herlyn M and Tuveson DA: Suppression of BRAF(V599E) in human melanoma abrogates transformation. Cancer Res 63: 5198-5202, 2003.

31. Freeman AK and Morrison DK: Mechanisms and potential therapies for acquired resistance to inhibitors targeting the Raf or MEK kinases in cancer. In: Molecular Mechanisms of Tumor Cell Resistance to Chemotherapy: Targeted Therapies to Reverse Resistance. Bonavita B (ed). pp47-67, 2013.

32. Prahallad A, Sun C, Huang S, Di Nicolantonio F, Salazar R, Zecchin D, Beijersbergen RL, Bardelli A and Bernards R: Unresponsiveness of colon cancer to BRAF(V600E) inhibition through feedback activation of EGFR. Nature 483: 100-103, 2012.

33. Van Schaeybroeck S, Karaiskou-McCaul A, Kelly D, Longley D, Galligan L, Van Cutsem E and Johnston P: Epidermal growth factor receptor activity determines response of colorectal cancer cells to gefitinib alone and in combination with chemotherapy. Clin Cancer Res 11: 7480-7489, 2005.

34. Li T, Ling YH, Goldman ID and Perez-Soler R: Scheduledependent cytotoxic synergism of pemetrexed and erlotinib in human non-small cell lung cancer cells. Clin Cancer Res 13: 3413-3422, 2007. 